\title{
POWER SYSTEM RESTORATION IN DISTRIBUTION NETWORK THROUGH RECONFIGURATION USING MST-KRUSKAL'S ALGORITHM
}

\author{
Pavana $\mathrm{D}^{\mathbf{1}}$, Triveni $\mathrm{M} \mathrm{T}^{\mathbf{2}}$ \\ ${ }^{1}$ Assistant Professor, Department of EEE, Bapuji Institute of Engineering \& Technology, Karnataka, India \\ ${ }^{2}$ Assistant Professor, Department of EEE, Bapuji Institute of Engineering \& Technology, Karnataka, India
}

\begin{abstract}
The proposed method provides one of the efficient management and operation of the distribution network through reconfiguration. Whenever an outage occurs in the distribution network, there may be partial or total block out of the system. In order to reduce interruptions to the consumers, proper switching of power lines is required and restoration of power as quickly as possible is essential. To reconfigure and determine optimal target network for minimization of the power losses, maximization of the load balance and for restoration of the power, is difficult task. The optimal target network is found through MST-Kruskal's (Minimum Spanning Tree) algorithm in IEEE standard 3 feeder, 16bus and 1 feeder, 33bus systems.
\end{abstract}

Keywords: Restoration of power, Spanning Tree, MST,

\section{INTRODUCTION}

Electrical energy is one of the basic need for the economic development of the country. In day today life, importance of electricity is more in the field of agriculture, industries, transportation facilities, commercial purpose etc. and supply of electricity to these sectors are taken for granted. Due to the complexity and natural factors of the electrical system, the faults occurring along the line is inherent or even greater to the system. Electrical distribution system plays a very important role in the power system network to meet consumers demand. Distribution network takes electricity from the transmission system and provides power to the consumers through branches. Depending upon the supply availability and reliability of overhead lines and underground cables are designed. Distribution system operates at low voltages levels, the losses are usually higher compared to those in other parts of the system. The careful designing, planning and installation with proper electrical equipment's is very important for economic considerations.

\subsection{Distribution System and its Outages}

The power distribution network delivers the electric power to the different types of loads and it has to supply the customers without any interruptions by concentrated on capacity issues and voltage tolerances. A typical distribution network consists of grids, feeder circuits, protective devices, primary and secondary circuits with distribution transformers, and service mains. It also contains number of switches like open switches and closed switches are respectively called as tie switches and sectionalizing switches. Outages occurs due to an unpredictable interruptions exist in a distribution system, it is very difficult to bring back the power system promptly to its initial state or to an optimal target network by switching operations. The problem of obtaining a target network by switching is called as power system restoration. The restored power supply to number of customers is as large and as quickly as possible, whenever an outage occurs. So many outages occurs due to different types of faults like forced and scheduled outages that exist in distribution system. Outages that occur due to sudden shutdown of the system in a network are called forced outages and scheduled outages are due to the maintenance fault. There are other outages that usually occur in distribution network are primary feeder outage, distribution transformer outage, and outages of the distribution lines[1].

\subsection{Restoration of Power in Distribution Network}

Whenever the fault occurs in distribution network, the power supply for both healthy and unhealthy components gets interrupted. The influence of faults on customers has to be reduced by proper switching of distribution power lines. Feeder loads in a distribution system are of different types. Individual load have different daily patterns that will occur in different times of the day which will make peak load (either lightly or heavily loaded) on the feeders. Reconfiguration of feeders in distribution network will make the redistribution of loads evenly. By opening or closing the status of sectionalizing and tie switches distribution network reconfigured [2]. To reduce voltage fluctuations, to maintain load balance, to minimize losses, to reduce switching time distribution system has to be reconfigured by considering some constraints such as radiality of the network, voltage drop, thermal capacity, current limits, restoration cost etc. To achieve these requirements shortest path or route is obtained by one of the graphical method called MSTKruskal's algorithm. 


\section{MST-KRUSKAL's (MINIMUM SPANNING}

\section{TREE) ALGORITHM}

In a graph theory, $\mathrm{T}$ is a connected, undirected spanning tree of the graph $\mathrm{G}$. That spanning tree is also a tree of the graph G. It consists of some of the lines and all the vertices of the graph G. That it doesn't forms any cycle or loop and every node lies in the tree. For the connected graph having $\mathrm{V}$ number of nodes, then $\mathrm{V}-1$ edges be there in spanning tree. For a connected, undirected graph spanning tree is sub graph of the main graph and contains all vertices without forming any loops. If the weight is assigned to each edge, each individual weight of the edges in a spanning tree decides its weight. For a particular spanning tree the total sum of weights of each line is its weight.

Minimum spanning tree or minimum weight spanning tree for a given undirected connected graph, is a spanning tree having a weight lesser or equal to other spanning tree in that graph.

MST algorithms are further classified into two types they are line based and node based algorithm. One of the algorithm (line based) that is proposed by MST approach is kruskal's algorithm in a graph theory, which finds a minimum spanning tree for a connected graph with weights. This algorithm forms a tree by determining the subset of the edges that includes every vertex, and the total weight of all the edges in the tree is minimized. If the given graph is not connected, then it finds a minimum spanning tree for each connected component [3]-[4].

To finds the MST by taking the smallest weight in the graph and connecting the two nodes and repeating until all nodes are connected to form a tree. This process starts with the line having minimum weight. This is done by assuming line impedance as its weights. Minimum spanning tree obtained by this algorithm contains every node of the algorithm, and doesn't forms any cycle or loop. MST obtained by this algorithm, the total weight of all the lines in that tree is minimized.

\subsection{Proposed Methodology}

The proposed method considers all of its laterals simultaneously, instead of determining the switching pattern on loop by loop basis. The buses and feeders are considered as nodes, distribution network impedance is considered as weight and distribution network line is considered as edges or lines. The total weights of all the edges in a graph are arranged in the ascending order. By these considerations the flowchart for kruskal's algorithm is as shown in fig 1 [5].

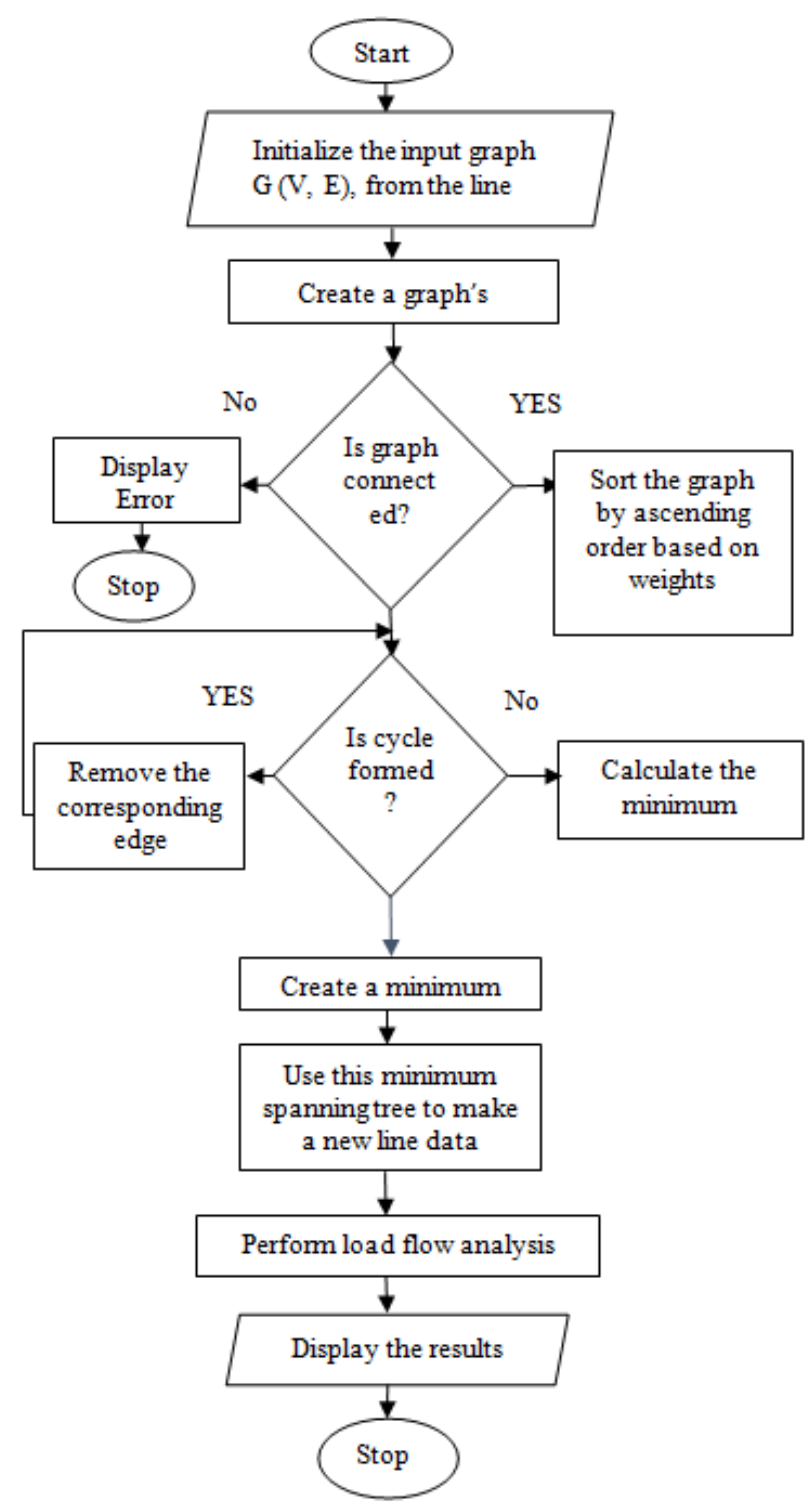

Fig-1: Flow chart for kruskal's algorithm

\section{IMPLEMENTATION OF PROPOSED}

\section{METHOD IN IEEE 3 FEEDER, 16 BUS AND 1 FEEDER, 33 BUS DISTRIBUTION SYSTEM}

Reconfiguration of distribution network using kruskal's algorithm is implemented using MATLAB software. In this work, considering the IEEE standard 3 feeder 16 bus, and 1 feeder 33 bus systems. N-R method is used to perform load flow analysis for these systems. The results are tabulated for both systems. Then kruskal's algorithm is applied to get MST (minimum impedance path), for this path load flow analysis is done.

\subsection{System 1: IEEE 3 Feeder, 16 Bus Radial System}

A standard IEEE 3 feeder, 16 buses radial distribution system is as shown in fig2, chosen as system1. Power to the system is fed through 3 feeders, designated as feeder 1 , 
feeder2, and feeder3. It has total 16 switches having 13 sectionalizing and 3 tie switches. It has 13 load center and different types of loads are connected. The system has base value 100MVA and base voltage of $20 \mathrm{kV}$.

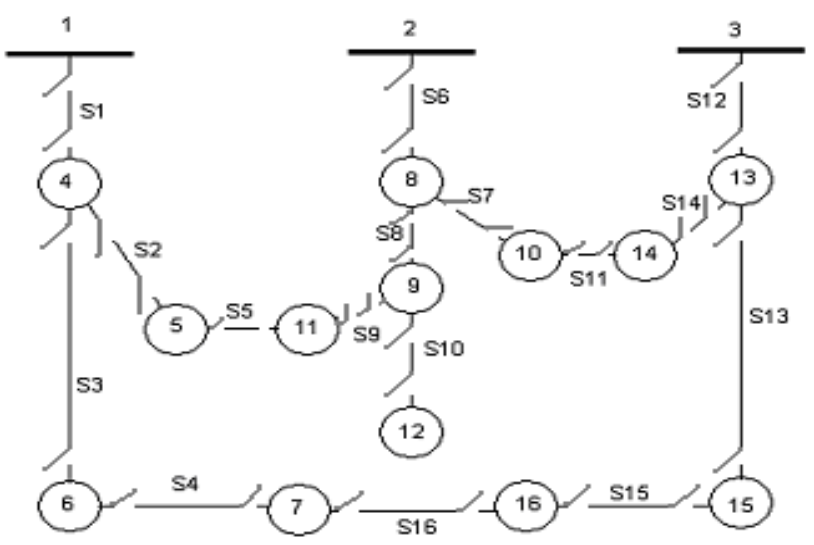

Fig-2: IEEE 3 feeder, 16 buses radial distribution system

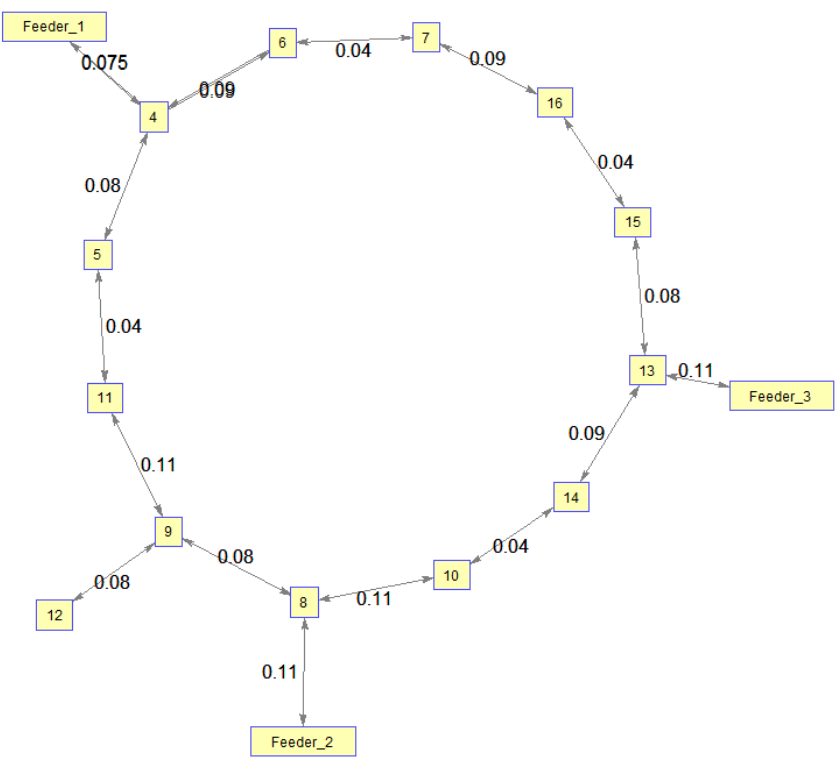

Fig-3: Graphical representation of 16 bus system
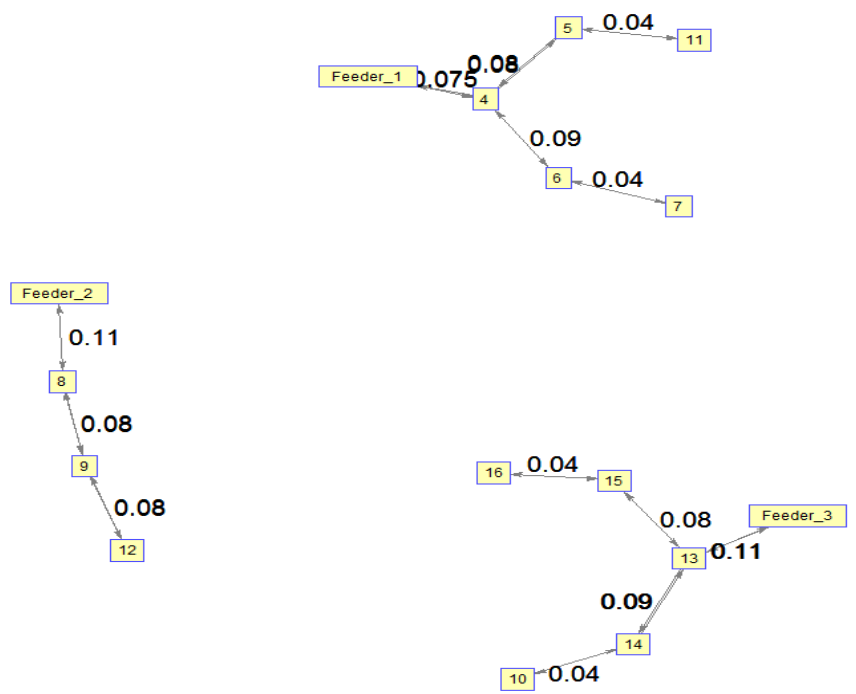

Fig-4: MST for3 feeder 16 bus system
A minimum impedance or shortest path is determined by implementing the kruskal's algorithm in MATLAB. The fig 3 shows graphical representation of 16 bus system with total weight of 1.265 . The total weight of the minimum impedance path is 0.73 . The shortest path for 16 bus system is shown fig4.

\subsection{System 2: IEEE 1 Feeder, 33 Bus Radial Distribution System}

IEEE 1 feeder, 33 bus is as shown in fig5 is considered as System2. It includes 33 nodes with a single feeder. Here the total numbers of sectionalizing switches are 33 and tie switches are 5.

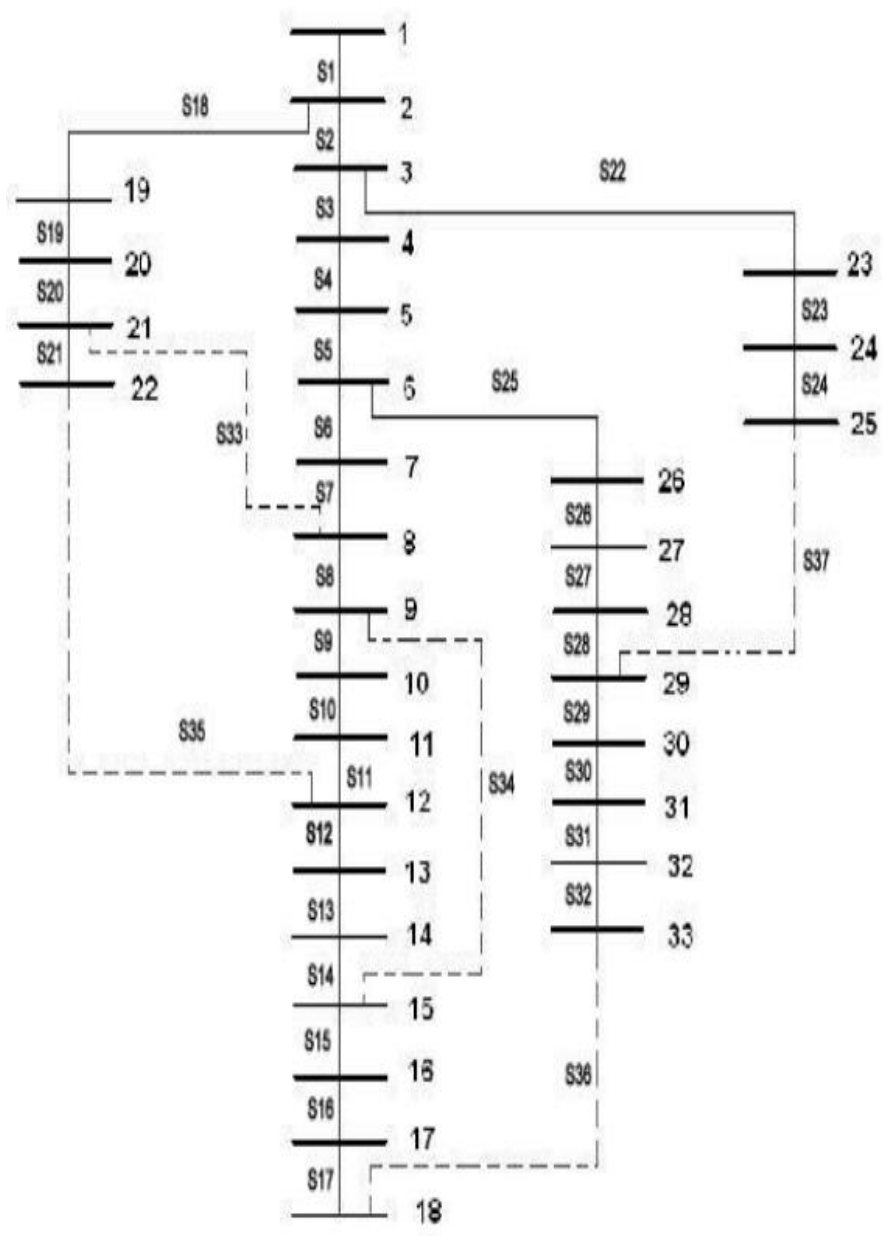

Fig -5: IEEE 1 feeder, 33 bus radial distribution system 


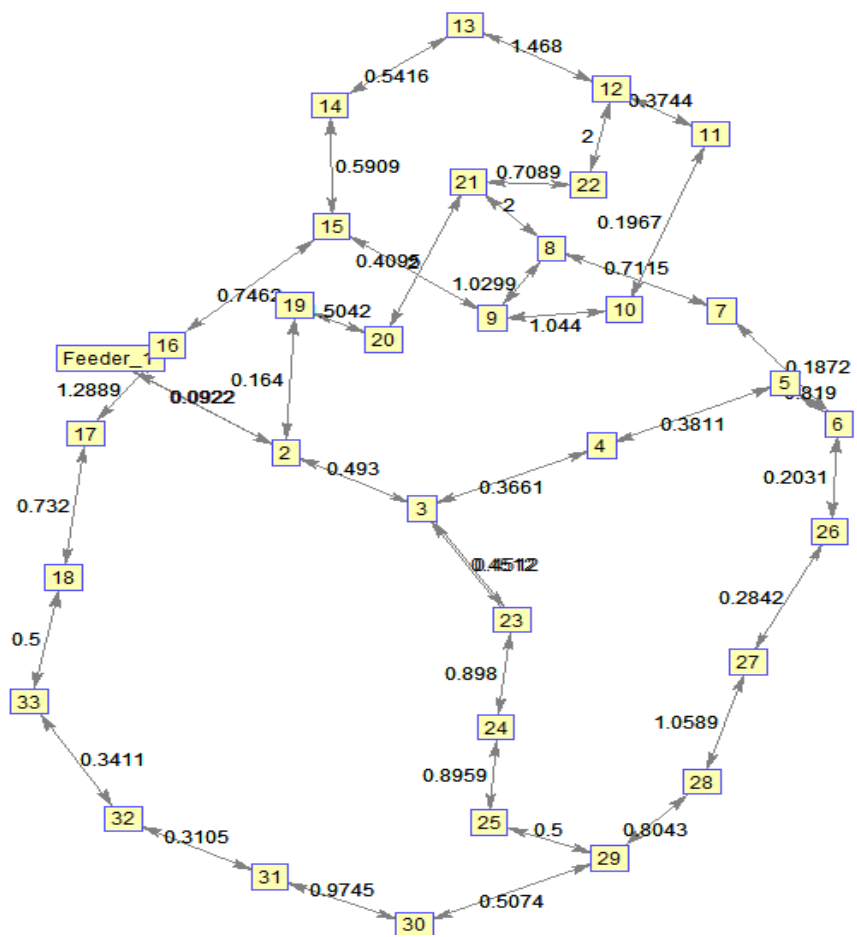

Fig-6: Graphical representation of 1 feeder, 33 bus system

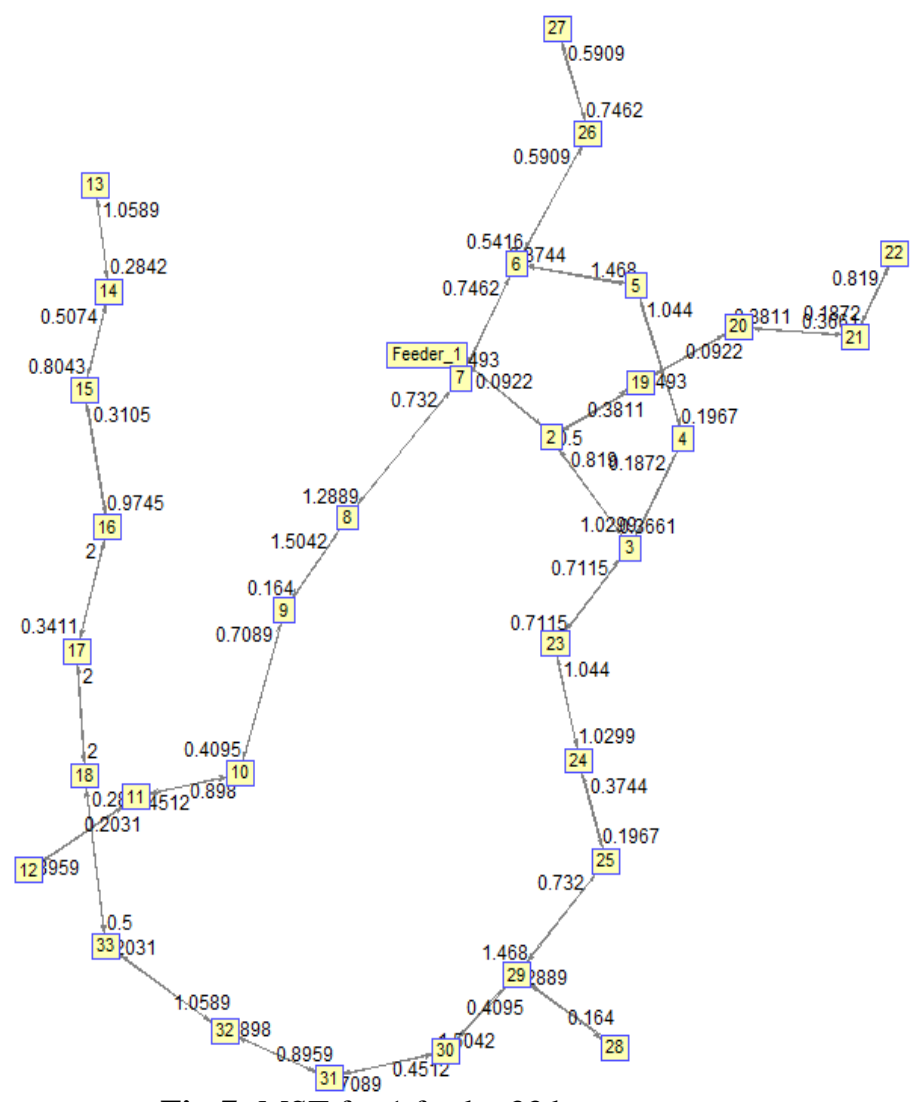

Fig-7: MST for 1 feeder 33 bus system

Graphical representation of 1 feeder, 33 bus system is as shown in the fig6, the total weight obtained is about 20.578 . A minimum impedance or shortest path, determined by implementing the Kruskal's algorithm is as shown in fig7. The total weight of the minimum impedance path is about 19.05 .

\section{RESULTS AND DISCUSSIONS}

The proposed scheme developed for both the systems is executed. The variation of load, generation, line losses and total weights for both bus systems with out and with kruskal's is as shown in table 1 and table 2 respectively.

Table-1: Without Kruskal's Algorithm

\begin{tabular}{|l|l|l|l|l|}
\hline \multirow{2}{*}{ BUS SYSTEM } & \multicolumn{3}{|l|}{$\begin{array}{l}\text { BUSS } \\
\text { SYSTEM }\end{array}$} & \multicolumn{2}{l}{ 33 } \\
& SYSTEM \\
\cline { 2 - 5 } & MW & MVAR & MW & MVAR \\
\hline LOAD & 28.7 & 5.9 & 3.175 & 2.3 \\
\hline GENERATION & 28.99 & 6.26 & 4.069 & 2.536 \\
\hline LINE LOSSES & 0.299 & 0.365 & 0.36 & 0.241 \\
\hline $\begin{array}{l}\text { TOTAL } \\
\text { WEIGHT }\end{array}$ & 1.265 & \multicolumn{2}{|l|}{20.578} \\
\hline
\end{tabular}

Table-2: With Kruskal's Algorithm

\begin{tabular}{|l|l|l|l|l|}
\hline \multirow{2}{*}{ BUS SYSTEM } & \multicolumn{2}{|l|}{$\begin{array}{l}\text { B } \\
\text { SYSTEM }\end{array}$} & \multicolumn{2}{l}{$\begin{array}{l}\text { S3 } \\
\text { SYSTEM }\end{array}$} \\
\cline { 2 - 5 } & MW & MVAR & MW & MVAR \\
\hline LOAD & 28.7 & 5.9 & 3.715 & 2.3 \\
\hline GENERATION & 28.68 & 5.916 & 3.716 & 2.3 \\
\hline LINE LOSSES & 0.126 & 0.157 & 0.27 & 0.194 \\
\hline $\begin{array}{l}\text { TOTAL } \\
\text { WEIGHT }\end{array}$ & 0.73 & & 19.065 & \\
\hline
\end{tabular}

Results obtained after calculation, it is found that for both bus systems before and after applying the kruskal's method reveals that,

- For 3 feeder, 16 bus system line losses are considerably reduces from $0.299 \mathrm{MW}$ to $0.126 \mathrm{MW}$ and 0.365 MVAR to 0.157 MVAR.

- For 1 feeder, 33 bus system, line losses are considerably reduces from $0.36 \mathrm{MW}$ to $0.27 \mathrm{MW}$ and $0.241 \mathrm{MVAR}$ to $0.194 \mathrm{MVAR}$.

\subsection{Simulation Outcome}

The graphs are plotted against voltage variations and bus number for both 3 feeder 16 and 1 feeder 33 bus systems.

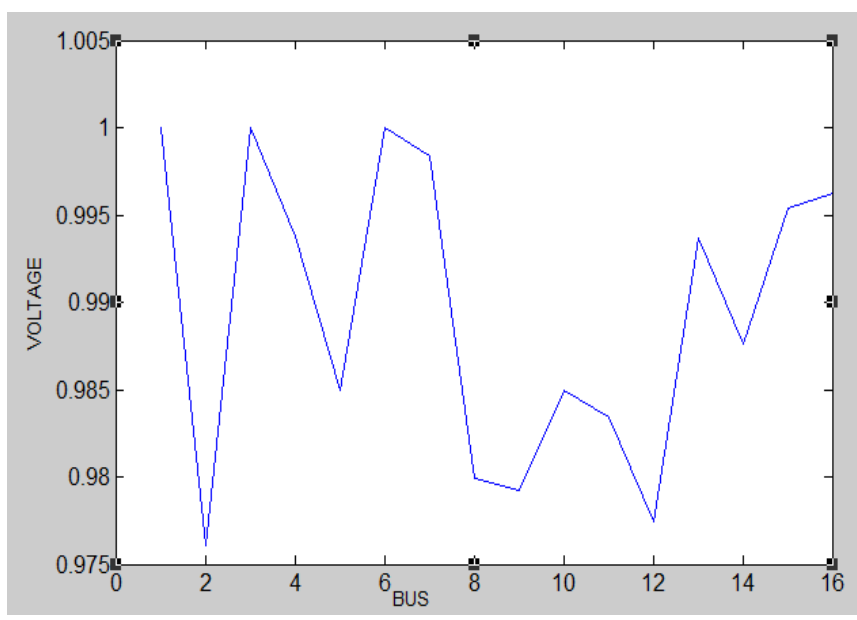

Fig-8 (a): Voltage magnitudes v/s buses for 3 feeders 16 bus system. 


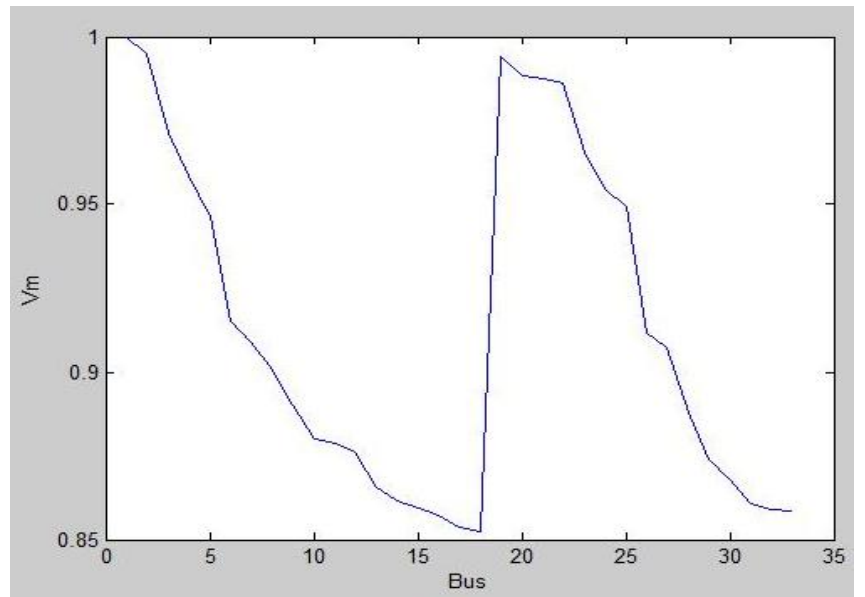

Fig-8(b): Voltage magnitude v/s buses for 1 feeder 33 bus system.

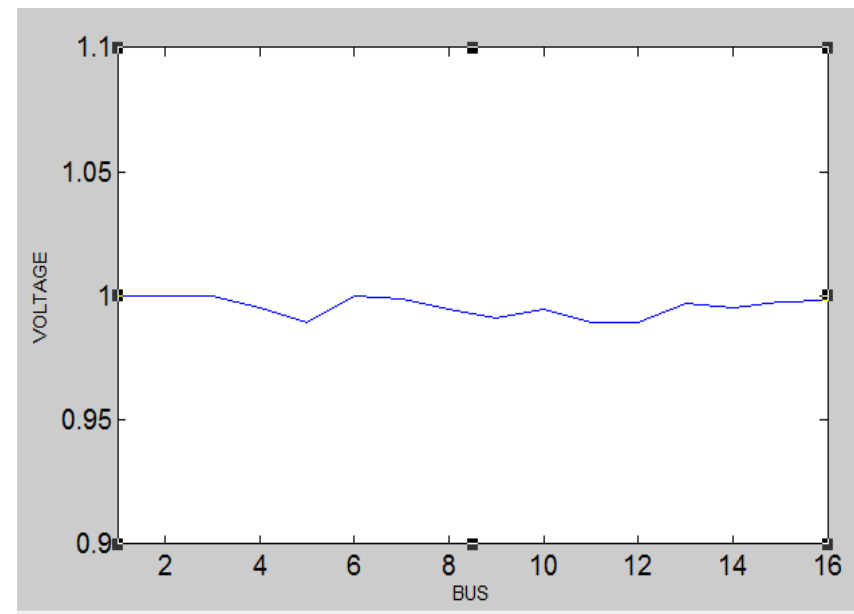

Fig-9(a): Voltage magnitudes v/s buses for 3 feeders 16 bus system after getting minimum impedance path.

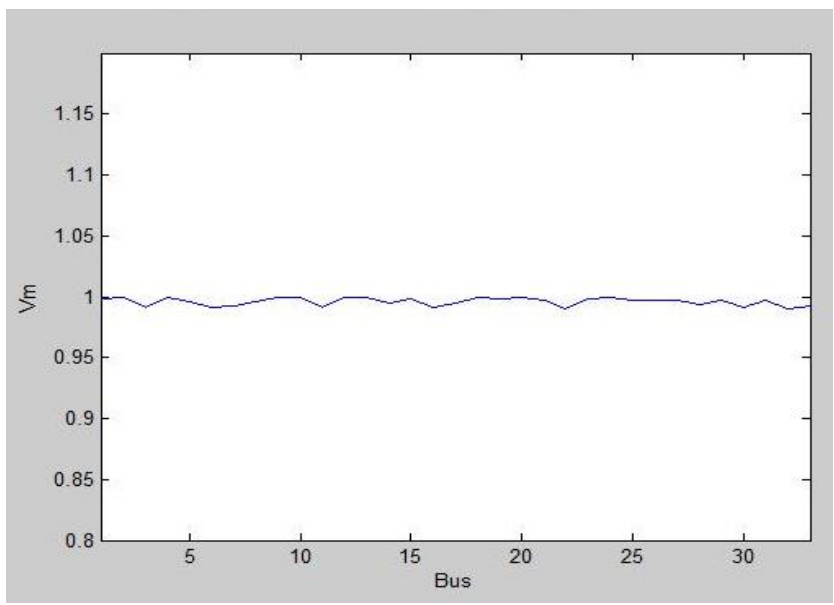

Fig-9(b): Voltage magnitudes v/s buses for 1 feeder 33 bus system after getting minimum impedance path.

Before applying kruskal's algorithm, observed that in fig 8(a) and fig 8(b) shows wide variations in voltage magnitude for both bus systems. Fig 9(a) and fig 9(b) shows voltage plot is almost near to 1 . That means it shows less variations and also losses are reduced because shortest path is easily obtained by the proposed algorithm. By observing the simulation analysis, it clearly shows that variation of voltage magnitude characteristics with respective buses is almost straight line. Loads are balanced and losses are also minimized.

\section{CONCLUSION}

With this methodology, percentage reduction in losses is found to be $17.3 \% \mathrm{MW}$ and $20.95 \% \mathrm{MVAR}$ and total weight is reduced from 1.265 to 0.73 for 3 feeder, 16 bus system. Similarly, for 1 feeder, 33 bus line losses are considerably reduced to $9 \% \mathrm{MW}$ and $4.7 \% \mathrm{MVAR}$ and also total weight is reduced from 20.578 to 19.065 . This methodology provides the way to calculate parameters of load and also provides the information about feeder over load condition without disturbing the existing network. This technique can be used to reconfiguration and restoring the power in distribution network.

\section{REFERENCES}

[1]. Different Techniques of Loss Minimization in Distribution System Gurpreet kour1 , R. K. Sharma2 1M. Tech Scholar, Electrical Engineering, Punjab, India er.gurpreetwazir@gmail.com

[2]. T. D. Sudhakar, "Power Restoration in Distribution Network Using MST Algorithms" New Frontiers in Graph Theory, Dr. Yagang Zhang (Ed.), ISBN: 978-953-51-01154, In Tech, Available from: http:/www.intechopen.com.

[3]. Sudhakar T D, et. Al, (2011), "Power System Restoration using Reverse Delete Algorithm Implemented in FPGA", Second IET International Conference on Sustainable Energy and Intelligent System, Page:373- 378.

[4]. Sudhakar T D, et. Al, (2010), "Prim's Algorithm for Loss Minimization and Service Restoration in Distribution Networks", International Journal of Electrical and Computer Engineering (IJEC), Volume 2, Number1 (2010), pp.43 62.

[5]. Sudhakar T D, et. Al, (2011), "Power System Reconfiguration based on Kruskal's Algorithm" IEEE conference ICEES 2011 Volume 1 Page No 234 - 240.

\section{BIOGRAPHIES}

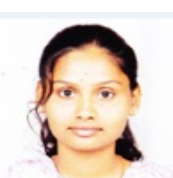

Pavana.D received her $\mathrm{BE}$ degree in the department of EEE, from VTU university, in 2012, M.Tech (Power systems) from VTU university in 2014, Karnataka, India. She is currently working as an Assistant Professor in the Dept. of EEE, BIET, Davangere, Karnataka, India.

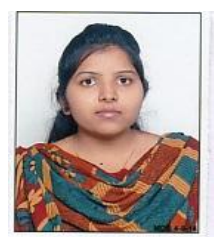

Triveni $\mathrm{M}$ T received her BE degree in the department of EEE, from Kuvempu university, Karnataka, in 2012, M.Tech (Computer Applications in Industrial Drives) from VTU university in 2014, Karnataka, India. She is currently working as an Assistant Professor in the Dept. of EEE, BIET, Davangere, Karnataka, India. 